\title{
Defective DNA repair systems and the development of breast and prostate cancer (Review)
}

\author{
YASUKO KITAGISHI ${ }^{*}$, MAYUMI KOBAYASHI and SATORU MATSUDA* \\ Department of Environmental Health Science, Nara Women's University, Nara 630-8506, Japan
}

Received September 14, 2012; Accepted October 29, 2012

DOI: 10.3892/ijo.2012.1696

\begin{abstract}
Genetic defects in DNA repair and DNA damage response genes often lead to an increase in cancer incidence. The role of defects is also associated with the modulation of hormone signaling pathways. A number of studies have suggested a role for estrogen in the regulation of DNA repair activity. Furthermore, mutations or epigenetic silencing in DNA repair genes have been associated with the sensitivity of cancers to hormonal therapy. The molecular basis for the progression of cancers from hormone-dependent to hormoneindependent remains a critical issue in the management of these types of cancer. In the present review, we aimed to summarize the function of DNA repair molecules from the viewpoint of carcinogenesis and hormone-related cell modulation, providing a comprehensive view of the molecular mechanisms by which hormones may exert their effects on the regulation of tumor progression.
\end{abstract}

\section{Contents}

1. Introduction

2. Association between hormones and DNA damage

3. Association between DNA damage and cancer therapy

4. Involvement of hormones and their receptors in DNA repair systems

5. Perspectives

\section{Introduction}

Breast and prostate cancers are known to be hormone-responsive types of cancer (1). There is evidence supporting the role

Correspondence to: Professor Satoru Matsuda, Department of Environmental Health Science, Nara Women's University, Kita-Uoya Nishimachi, Nara 630-8506, Japan

E-mail: smatsuda@cc.nara-wu.ac.jp

*Contributed equally

Key words: DNA repair, estrogen, androgen, p53, BRCA1, carcinogenesis of hormones in stimulating cancer cell growth. In addition, the progression from hormone-dependent to hormone-independent disease remains a critical issue in the management of these cancers. Recently, studies have reported an association between DNA repair deficiency and loss of hormone receptors (2). The modulation of the DNA repair system may contribute to hormone-independent cancer development. There may be connections between carcinogenesis, the hormone endocrine system and DNA repair systems (Fig. 1). It has been suggested that the immune system surveys the body for cancerous cells, inhibiting carcinogenesis.

Cancer is a complex disease which can aquire a more invasive and therapy-resistant character by a number of molecular changes. The malignant transformation occurs in the cells with increased proliferative activity since DNA replication errors accumulate along with the high DNA duplication rate (3-5). However, cells possess a machinery to maintain genomic integrity in response to various genotoxic events. Under genotoxic conditions, cells do not progress through the cell cycle by activating the DNA damage checkpoint (6-8). The checkpoint acts as a process to transmit information from damaged DNA lesions to the cell cycle regulators. Mutations or downregulations in several genes which make the effects of DNA damage less severe are known to predispose to cancer development. For example, mutations in ataxia telangiectasia-mutated (ATM) have been associated with an increased risk of cancer $(9,10)$. The ATM is a checkpoint kinase that phosphorylates a number of proteins in response to DNA damage, including the tumor suppressor gene products, p53 and BRCA1, which have been implicated in DNA repair pathways. Normal cells show a balance of the various mechanisms of the DNA repair machinery. The p53 tumor suppressor regulates several DNA repair pathways involved in the cellular response to genotoxic stresses, which can block cell proliferation and/or induce apoptosis (11). Since p53 also plays an important role in the transcriptional regulation of genes encoding proteins involved in DNA repair and apoptosis, the modification of p53 may appear to be a pivotal determinant of the fate of cells. During carcinogenic progression, $p 53$ is mutated or deleted in almost half of all human cancers $(12,13)$ and fails to function normally. $B R C A 1$ regulates the expression of several genes identified as having key roles in breast and ovarian cancer risk. BRCAI also participates in DNA repair and recombination processes related to the maintenance of genomic integrity and the control of cell proliferation (14). As most BRCAl mutant cancers are 
hormone receptor-negative, hormonal factors may contribute to the etiology of BRCA1 mutant cancers $(15,16)$.

Advances in the field of DNA repair biology have led to a better understanding of the molecular events which are related to the pathogenesis of hormone-related cancers. Indeed, an understanding of the underlying molecular mechanisms involved in the transition to hormone refractory disease is also essential for the development of effective therapeutic and preventive strategies. In the present review, we summarize the function of DNA repair molecules from the viewpoint of carcinogenesis and hormone-related cell modulation.

\section{Association between hormones and DNA damage}

Since estrogens stimulate cell proliferation (17), increased exposure to estrogens promotes the opportunity to develop random genetic errors. In addition, some hormone oxidative metabolites catalyzed by cytochrome p450 enzymes can form unstable adducts with DNA leading to mutations (18). Furthermore, the metabolic process produces reactive oxygen species that accounts for oxidative damage to genome DNA. The continuous mutagenic potential may contribute to the initiation of cancer. Prolonged exposure to estrogen is strongly associated with an increased risk of developing breast cancer. Estrogens bind primarily to estrogen receptors, which are believed to mediate the various actions of estrogens. On the other hand, androgen signaling may induce apoptosis by activating the DNA damage response (19). The BRCA1 protein may function to suppress mammary epithelial cell proliferation by inhibiting estrogen receptor-mediated pathways (Fig. 2). In addition, an estrogen receptor complex modulates BRCA1 transcription under conditions of estrogen stimulation. Recent studies have shown an association between DNA repair deficiency and loss of estrogen as well as androgen receptors (2). In addition, estrogens are not only essential for mammary growth and differentiation, but also enhance the activity of the p53 tumor suppressor protein (20). This paradox, the bidirectional functions of some hormones in carcinogenesis or anticarcinogenesis, should be resolved in the near future.

p53 is a key transcription factor that regulates several genes and protects against genomic instability. Mutations in the $p 53$ gene are frequent in breast cancers and are associated with poor prognosis. The focal adhesion kinase (FAK), a tyrosine kinase, is overexpressed in a variety of human tumors including breast cancers (21). FAK is a critical regulator of cell motility and its overexpression is associated with increased metastatic potential. FAK expression is downregulated in a p53-dependent manner in response to estrogen (22). The loss of FAK downregulation in p53 mutant cells correlates with increased invasive capacity upon estrogen stimulation. In this way, p53 is an important downregulator of FAK and the loss of p53 function in breast cancer may contribute to the metastatic potential of estrogen-responsive cancers. The p53 downstream transcriptional target, p21/WAF-1, is increased in estrogentreated cells in response to DNA damage. The $p 53$ gene may form a center within a network connected by genes that are regulated by hormones and DNA repair. In addition, the crosstalk between p53 and androgen receptor signaling suggests that p53 activation may augment the anticancer effects of hormonal therapy in prostate cancer (23).

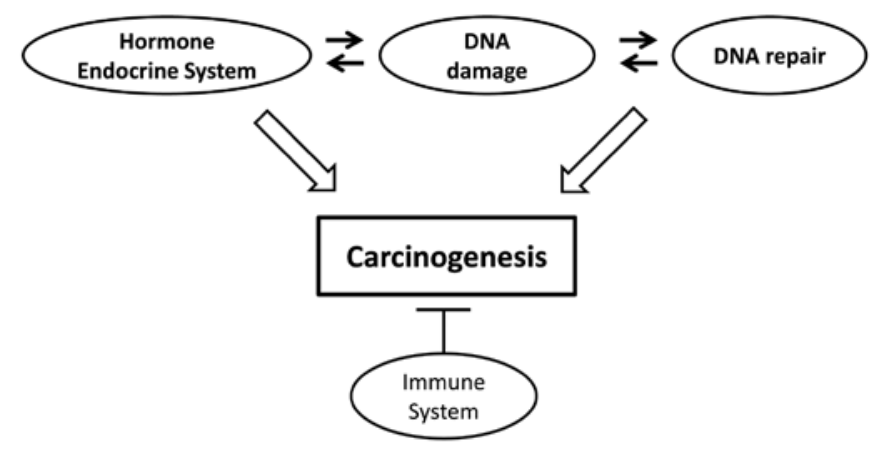

Figure 1. Involvement of the hormone endocrine, immune, DNA damage and DNA repair systems in cancer. The relevance of DNA damage and DNA repair to carcinogenesis has become evident. Hormones may be a causative factor in the development of specific types of cancer. There is an association between hormones and DNA repair activity. A challenge for the immune system is to recognize and eliminate cells undergoing carcinogenesis.

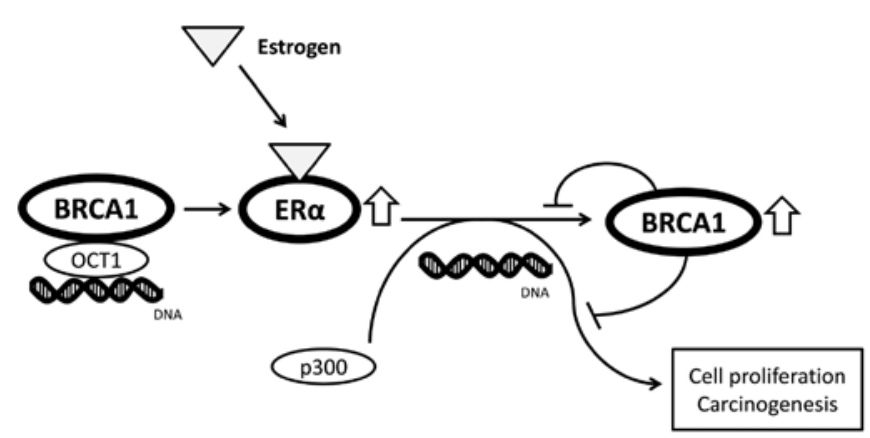

Figure 2. Schematic representation of the regulatory interplay between BRCA1 and estrogen receptor (ER)- $\alpha$. BRCA1 regulates the expression of ER- $\alpha$ in a transcription factor (OCT1)-dependent manner. Estrogen stimulation increases the expression of BRCA1 through the ER- $\alpha$ transcriptional mechanism involving the transcriptional co-activator, p300. In addition, the BRCA1 protein can negatively regulate the ER- $\alpha$-mediated transactivation of downstream genes. Note that some critical events have been omitted for clarity.

The combined ingestion of soy isoflavone and curcumin decreases the serum level of prostate-specific antigen (PSA) (24). This combination affects the expression and phosphorylation of ATM and inhibits prostate cancer cell proliferation. Testosterone, an androgen, augments the activation of the DNA damage-response, which may suppress the initial malignant transformation of prostate cancer. Vitamin D has also been shown to exhibit cancer-preventive activities. There is evidence that vitamin D provides protection against DNA damage (25). Vitamin D enhances the expression of DNA repair genes, such as ATM and Rad50, thereby promoting effective DNA doublestrand break (DSB) repair, protecting cells from DNA damage and stress and consequently, carcinogenesis (26).

\section{Association between DNA damage and cancer therapy}

Exposure to ionizing radiation or DNA-damaging agents causes DSBs. Genetic defects in DNA damage response genes and/or downregulation of the DNA repair mechanism promote genomic instability, which can lead to carcinogenesis (27). Cells 


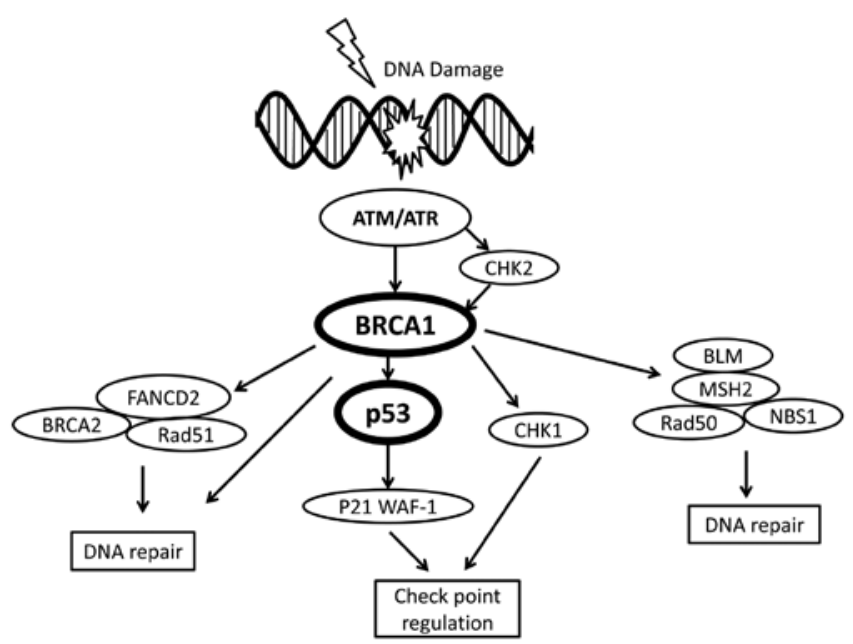

Figure 3. Schematic representation and overview of the DNA repair and checkpoint regulation of cell cycle signaling pathways. Examples of the molecules known to act on regulatory pathways are shown. Note that some critical pathways have been omitted for clarity.

are equipped with multiple DNA repair mechanisms for the maintenance of genomic stability and the suppression of carcinogenesis. Standard DNA repair pathways available in mammalian cells include homologous repair, non-homologous end-joining and single-strand annealing (28). There are different pathways that repair DSBs. The DNA repair system is essential for the survival of normal and cancer cells. A set of signaling pathways detects the DSBs and mediates either DNA repair survival or apoptotic cell death. The main DNA damage recognition molecule is ATM (29), which is a checkpoint kinase that phosphorylates a number of proteins in response to DNA damage, including p53 and BRCA1 (Fig. 3). Schematic structures of these important molecules are shown in Fig. 4. An additional consequence of defective DNA repair is cellular hypersensitivity to DNA-damaging agents (30). The inhibition of DNA repair pathways blocks the mechanisms that are required for survival in the presence of oncogenic mutations. DNA-damaging agents then function more effectively as a therapeutic strategy for cancer cells with DNA repair defects (30). Epigenetic mechanisms, such as histone modifications and DNA methylation have been evaluated with a view of enhancing cancer therapy via the regulation of the expression of genes involved in DNA repair (31).

Through stress-induced activation, p53 triggers the expression of the target genes that protect the genetic integrity of cells. The p53 gene is frequently mutated in multiple cancer tissues, suggesting that p53 plays a critical role in preventing cancers. Mutant $p 53$ can be classified as a loss-of-function or gain-of-function protein depending on the mutation type (32). Wild-type p53 is inactive under normal physiological conditions and is activated in response to various types of DNA-damaging stresses. p53 activation may lead to the regression of existing neoplastic lesions and may therefore be important in developing cancer prevention (33). Failure of the DNA repair functions leads to p53-mediated induction of apoptotic cell death. The p53 protein undergoes post-translational modifications such as acetylation of lysines, nitration of tyrosines and the phosphorylation of serine/threonine residues in response to certain stresses (34). In addition, multiple mechanisms have been revealed to regulate p53 activity, which determines the selectivity of p53 for specific transcriptional targets. Among the transcriptional targets of p53, p21WAF-1 has been shown to play an important role in both p53-dependent (35) and -independent pathways (36). p21WAF-1 inhibits cell cycle progression through the interaction with the cyclin-dependent kinase (CDK) complex. In this way, p53 has been known to

\begin{tabular}{|c|c|c|c|c|}
\hline $\begin{array}{c}\text { ATM } \\
1\end{array}$ & (D) & (P) (P) & & 3056 \\
\hline - & HEAT repeats & FAT & PI3-K & FATC \\
\hline
\end{tabular}
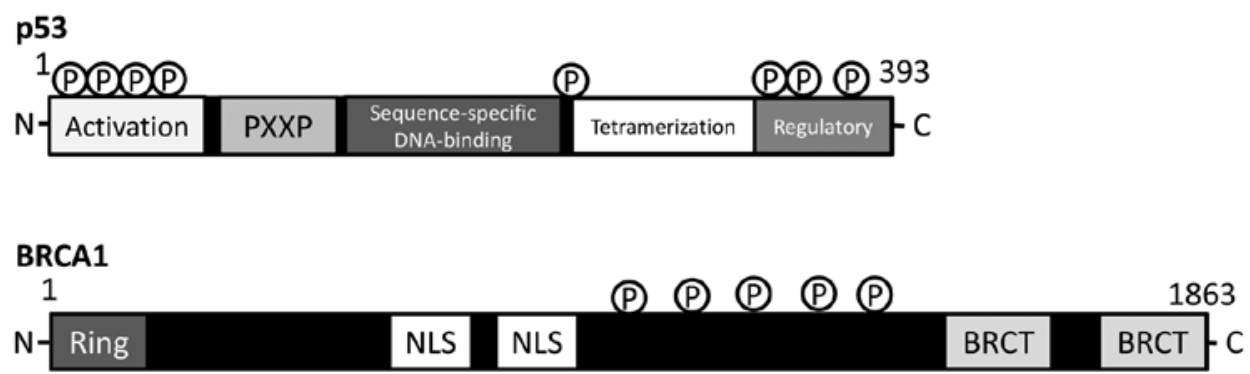

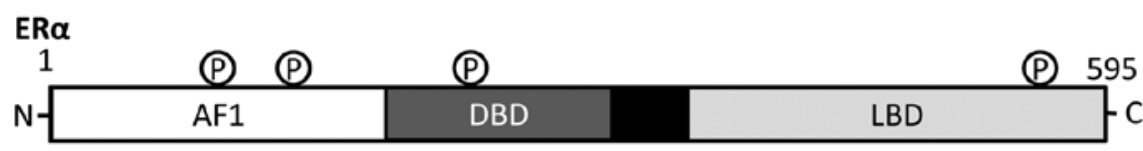

Figure 4. Schematic diagram indicating the domain structures of ATM, p53, BRCA1 and estrogen receptor (ER)- $\alpha$ proteins. The functionally important sites including the sites of protein phosphorylation are shown. Note that the sizes of proteins are modified for clarity. HEAT, huntington, elongation factor 3 , a subunit of PP2A and TOR1; FAT, FRAP-ATM-TRRAP; FATC, FAT-C-terminal; PXXP, a proline repeat, Ring, really interesting new gene, finger domain; NLS, nuclear localization signal, BRCT, BRCA1 C terminus; AF1, activation function 1 DBD, DNA-binding domain; LBD, ligand-binding domain. 
play a central role in maintaining a stable genome through its role in cell cycle checkpoints, DNA repair and apoptosis.

BRCA1 also fulfills the criteria for a tumor suppressor gene whose function is required to block cancer development. BRCAI hereditary breast cancer is a type of cancer with defects in a DNA repair pathway (37). The mutation is then associated with increased genomic instability in cells, which accelerates the mutation rate of other critical genes. Studies have established functional roles for BRCA1 in DNA damage signaling, DNA repair processes and cell cycle checkpoints (38). Consistent with these functional roles, cells deficient in BRCAl exhibit severe genomic instability and chromosomal aberrations. Although BRCAl gene mutations are rare in sporadic breast and ovarian cancers, BRCA1 protein expression is frequently reduced in sporadic cases (39). BRCAl cDNA encodes for the 1863-amino acid protein with an amino terminal zinc ring finger motif and two putative nuclear localization signals (Fig. 4). The aminoterminal domain possesses E3 ubiquitin ligase activity (40) and the carboxyl-terminal domain is involved in binding to specific phospho-proteins (41). The role of BRCA1 in cell cycle control involves its ability to interact with various cyclins and CDKs, activate the CDK inhibitor, p21WAF-1, and p53. BRCA1 becomes hyperphosphorylated following exposure to DNA-damaging agents, and the specific function of BRCA1 is regulated by phosphorylation (42). In addition to the roles in the regulation of DNA damage response, the BRCA1 protein interacts with the estrogen and androgen receptor, and regulates their activity (43), inhibiting estrogen receptor- $\alpha$ activity and stimulating androgen receptor activity. Thus, BRCAl mutations confer a modulatory risk for each type of hormone-responsive cancer. In other words, hormonal factors intensely contribute to the cancer risk in BRCA1 mutation carriers. Alternatively, hormonal factors also affect therapeutic tumor cell killing via modulating DNA repair. Hence, the DNA repair capacity may be a novel therapeutic modality in overcoming drug resistance in cancer. Either survival or apoptosis, which is determined by the balance between DNA damage and DNA repair capacity, may thus be one of the major problems of cancer therapy.

\section{Involvement of hormones and their receptors in DNA repair systems}

Hormones, such as estrogen, progesterone and androgen often contribute to the initiation and promotion of carcinogenesis via specific hormone receptors. A number of candidate genes have been identified as biomarkers for breast and prostate cancers such as those involved in hormone-synthesis, activation and secretion (44). Furthermore, hormonal therapies generally regulate cancer cell growth and have provided improvements in survival in hormone-related diseases. For example, anti-estrogens are effective in estrogen receptor-positive breast cancer, and androgen deprivation therapies are common in advanced prostate cancer. However, transition to hormone refractory cancer cells remains an important clinical issue, which limits the benefits of these therapies.

Estrogens, not only play a key role in the proliferation and differentiation of the mammary epithelium, but also regulate diverse cellular processes in brain, cardiovascular and bone metabolism (45). In addition, several normal and carcinoma cells express estrogen receptors and their proliferation is stimulated by estrogen, which in turn increases the likelihood that DNA damage occurs. The chronic exposure estrogen then contributes to carcinogenesis in estrogen receptor-positive cells. However, estrogens also stimulate the expression of genes that can repair DNA damage including BRCA1 (Fig. 2). DNA repair defects in the progression of breast cancer may occur in the transition to hormone-independence. The estrogen-bound receptor dimerizes and associates with chromatin. The receptor dimers bind directly to a DNA sequence motif, the estrogen response element. There are two, estrogen receptor- $\alpha$ and estrogen receptor- $\beta$. Estrogen receptor- $\alpha$ is thought to be proliferative, whereas the activation of estrogen receptor- $\beta$ is thought to induce apoptosis. A key DNA repair protein, $\mathrm{MSH} 2$, has been shown to be a potent co-activator of estrogen receptor- $\alpha$ (46). An increase in estrogen receptor- $\beta$ levels may be associated with a reduction in breast cancer risk (47). There is evidence showing that the reduced expression of estrogen receptor- $\beta$ correlates with increased prostate cancer risk. Estrogen receptor- $\beta$ may inhibit cellular proliferation by antagonizing the actions of estrogen receptor- $\alpha$ (48). Exposure to genistein, which is an estrogen-like chemical compound present in plants such as soy, reduces breast cancer risk (49). The expression of BRCA1 is upregulated in mammary glands prepubertally exposed to genistein, suggesting that BRCA1 plays a role in mediating the cancer-protective effects of genistein. However, high soy intake does not reduce breast cancer risk if consumed in adulthood. In addition, physiologically high levels of estrogen are believed to stimulate carcinogenesis. Childhood and adolescence may be particularly sensitive periods for breast cancer initiation. There is an urgent need to further understand DNA repair pathways and their distinct roles in childhood and adulthood.

Prostate cancer is a frequently diagnosed ( 20\%) malignancy in males $(50,51)$. The risk factors may act through hormonal mechanisms, since the drug that blocks androgenic hormone activation reduces cancer risk. In addition, some sporadic prostate cancer cases are linked to polymorphisms of genes involved in androgen activation and its signaling pathway $(50,51)$. Androgens have been recognized to play a role in controlling the growth of the normal prostate gland. The androgen receptor is a ligand-activated transcription factor of the nuclear receptor superfamily that plays a critical role in male physiology, whose signaling also contributes to carcinogenesis and cancer progression by regulating the transcription of androgen-responsive genes. One of the androgen-responsive genes, PSA, is a clinically important marker that is used for the diagnosis and evaluation of the prostate cancer (52). During the progression of androgensensitive prostate cancer to androgen-refractory, the majority of cancer cells still express the androgen receptor; however, in many cases it is mutated. The downregulation of the androgen receptor induces apoptotic cell death and inhibits cancer cell proliferation. Therefore, androgen signaling has been recognized as a key target for prostate cancer prevention and therapy.

Variations in estrogen receptor activities may also modulate prostate cancer risk (53). The increased consumption of phytoestrogens such as genistein, also a well-known tyrosine kinase inhibitor, has been associated with a reduced risk of prostate cancer (54). Phytoestrogens protect cells against reactive oxygen species by scavenging free radicals. In addition, phytoestrogens upregulate the expression of glycogen synthase kinase-3 $\beta$ (GSK$3 \beta$ ), enhance GSK-3 $\beta$ binding to $\beta$-catenin, and induce apoptotic 
cell death (55), suggesting that phytoestrogens can induce apoptosis and inhibit prostate cancer cell growth. Phytoestrogens have also been found to inhibit molecules in the mitogen-activated kinase (MAPK) pathway (56). Furthermore, they can block the activation of p38-MAPK by TGF- $\beta$ in prostate cancer (57), inhibiting cancer cell invasion and metastasis. Reduced MSH2 protein expression in prostate cancer cells has been shown to correlate with an overall recurrence-free interval, while the reduced protein expression may be associated with an increased risk of prostate cancer initiation (58). MSH2 expression has been shown to correlate with the expression of $\mathrm{p} 53$, while it negatively correlates with the expression of the estrogen receptor (59). The downregulation of the $\mathrm{MSH} 2$ gene has been associated with invasive breast cancer and the hormone independence of prostate cancer. Elevated post-meiotic segregation increased 2 (PMS2) expression also appears to negatively correlate with prognosis in prostate cancer patients $(60,61)$. The overexpression of PMS2 may confer DNA damage tolerance.

\section{Perspectives}

The hormone signaling pathway is a complex signaling network, and further in-depth research in this area is required. The DNA repair system is a highly conserved DNA editing process that maintains genomic fidelity through the recognition and repair of damaged nucleotides. Discrepancy between cell proliferation and DNA repair functions may account for the accumulation of DNA errors and vulnerability to carcinogenesis. The roles of human tumor susceptibility genes in the DNA repair machinery are important for the development of methods for the prediction of risk, diagnosis, prevention and therapy of human cancers. For example, as hormonal regulation contributes to the expression of the tumor susceptibility gene product, BRCA1, the hormonal prevention of $B R C A 1$ mutant breast cancers may be effective. The discovery of other molecular pathways would be helpful to further understand the fundamental mechanisms of hormone independence. Certain evidence supports the cancer-protective roles of dietary selenium, vitamin D, vitamin E, lycopene and soy foods $(62,63)$. Lycopene, which is a carotenoid found in tomatoes, appears to be an effective protective dietary factor for prostate cancer, and acts as an antioxidant by scavenging reactive oxygen species to protect against DNA damage. A significantly reduced risk of prostate cancer following higher lycopene consumption has been shown. Further mechanistic studies are also required in order to understand the precise molecular mechanisms of hormonal carcinogenesis, cancer prevention and the DNA repair system for more effective therapeutic interventions of these human malignancies.

\section{Acknowledgements}

This study was supported by Grants-in-Aid from the Ministry of Education, Culture, Sports, Science and Technology of Japan. In addition, this study was supported in part by a grant from Shin-ei Pharmaceutical Co., Ltd.

\section{References}

1. Folkerd EJ and Dowsett M: Influence of sex hormones on cancer progression. J Clin Oncol 28: 4038-4044, 2010.
2. Martin L, Coffey M, Lawler M, Hollywood D and Marignol L: DNA mismatch repair and the transition to hormone independence in breast and prostate cancer. Cancer Lett 291: 142-149, 2010.

3. Xu Y and Price BD: Chromatin dynamics and the repair of DNA double strand breaks. Cell Cycle 10: 261-267, 2011.

4. Crasta K, Ganem NJ, Dagher R, Lantermann AB, Ivanova EV, Pan Y, et al: DNA breaks and chromosome pulverization from errors in mitosis. Nature 482: 53-58, 2012.

5. Rodriguez GP, Romanova NV, Bao G, Rouf NC, Kow YW and Crouse GF: Mismatch repair-dependent mutagenesis in nondividing cells. Proc Natl Acad Sci USA 109: 6153-6158, 2012

6. Vurusaner B, Poli G and Basaga H: Tumor suppressor genes and ROS: complex networks of interactions. Free Radic Biol Med 52: 7-18, 2012.

7. Hanel $\mathrm{W}$ and Moll UM: Links between mutant $\mathrm{p} 53$ and genomic instability. J Cell Biochem 113: 433-439, 2012.

8. Jones RM and Petermann E: Replication fork dynamics and the DNA damage response. Biochem J 443: 13-26, 2012.

9. Smith J, Tho LM, Xu N and Gillespie DA: The ATM-Chk2 and ATR-Chk1 pathways in DNA damage signaling and cancer Adv Cancer Res 108: 73-112, 2010.

10. Hennequin C, Quero L and Favaudon V: DNA repair and tumour radiosensitivity: focus on ATM gene. Bull Cancer 98: 239-246, 2011.

11. Molchadsky A, Rivlin N, Brosh R, Rotter V and Sarig R: p53 is balancing development, differentiation and de-differentiation to assure cancer prevention. Carcinogenesis 31: 1501-1508, 2010.

12. Muller PA, Vousden KH and Norman JC: p53 and its mutants in tumor cell migration and invasion. J Cell Biol 192: 209-218, 2011.

13. Essmann $\mathrm{F}$ and Schulze-Osthoff $\mathrm{K}$ : Translational approaches targeting the p53 pathway for anti-cancer therapy. Br J Pharmacol 165: 328-344, 2012.

14. Karve TM, Li X and Saha T: BRCA1-mediated signaling pathways in ovarian carcinogenesis. Funct Integr Genomics 12: 63-79, 2012.

15. Berstein LM: Endocrinology of the wild and mutant BRCA1 gene and types of hormonal carcinogenesis. Future Oncol 4: 23-39, 2008.

16. Okumura N, Yoshida H, Kitagishi Y, Nishimura Y and Matsuda S: Alternative splicings on p53, BRCA1 and PTEN genes involved in breast cancer. Biochem Biophys Res Commun 413: 395-399, 2011.

17. Mueck AO and Sitruk-Ware R: Nomegestrol acetate, a novel progestogen for oral contraception. Steroids 76: 531-539, 2011.

18. Bolton JL and Thatcher GR: Potential mechanisms of estrogen quinone carcinogenesis. Chem Res Toxicol 21: 93-101, 2008.

19. Comstock CE and Knudsen KE: The complex role of $A R$ signaling after cytotoxic insult: implications for cell-cyclebased chemotherapeutics. Cell Cycle 6: 1307-1313, 2007.

20. Lu S, Becker KA, Hagen MJ, Yan H, Roberts AL, Mathews LA, et al: Transcriptional responses to estrogen and progesterone in mammary gland identify networks regulating p53 activity. Endocrinology 149: 4809-4820, 2008.

21. Golubovskaya VM, Conway-Dorsey K, Edmiston SN, Tse CK, Lark AA, Livasy CA, et al: FAK overexpression and p53 mutations are highly correlated in human breast cancer. Int $\mathrm{J}$ Cancer 125: 1735-1738, 2009.

22. Anaganti S, Fernández-Cuesta L, Langerød A, Hainaut $P$ and Olivier M: p53-Dependent repression of focal adhesion kinase in response to estradiol in breast cancer cell-lines. Cancer Lett 300: 215-224, 2011.

23. Lee HJ, Chattopadhyay S, Yoon WH, Bahk JY, Kim TH, Kang HS, et al: Overexpression of hepatocyte nuclear factor-3alpha induces apoptosis through the upregulation and accumulation of cytoplasmic p53 in prostate cancer cells. Prostate 70: 353-361, 2010.

24. Ide H, Tokiwa S, Sakamaki K, Nishio K, Isotani S, Muto S, et al: Combined inhibitory effects of soy isoflavones and curcumin on the production of prostate-specific antigen. Prostate 70: 1127-1133, 2010.

25. Welsh J: Cellular and molecular effects of vitamin D on carcinogenesis. Arch Biochem Biophys 523: 107-114, 2012.

26. Ting HJ, Yasmin-Karim S, Yan SJ, Hsu JW, Lin TH, Zeng W, et al: A positive feedback signaling loop between ATM and the vitamin $\mathrm{D}$ receptor is critical for cancer chemoprevention by vitamin D. Cancer Res 72: 958-968, 2012.

27. Kelly GL and Strasser A: The essential role of evasion from cell death in cancer. Adv Cancer Res 111: 39-96, 2011. 
28. Natarajan AT and Palitti F: DNA repair and chromosomal alterations. Mutat Res 657: 3-7, 2008.

29. Bhatti S, Kozlov S, Farooqi AA, Naqi A, Lavin M and Khanna KK: ATM protein kinase: the linchpin of cellular defenses to stress. Cell Mol Life Sci 68: 2977-3006, 2011.

30. Okumura N, Yoshida H, Kitagishi Y, Nishimura Y, Iseki S and Matsuda S: Against lung cancer cells: to be, or not to be, that is the problem. Lung Cancer Int: doi:10.1155/2012/659365, 2012.

31. Gigek CO, Chen ES, Calcagno DQ, Wisnieski F, Burbano RR and Smith MA: Epigenetic mechanisms in gastric cancer. Epigenomics 4: 279-294, 2012.

32. Martinez-Rivera M and Siddik ZH: Resistance and gain-ofresistance phenotypes in cancers harboring wild-type p53. Biochem Pharmacol 83: 1049-1062, 2012.

33. Stegh AH: Targeting the p53 signaling pathway in cancer therapy - the promises, challenges and perils. Expert Opin Ther Targets 16: 67-83, 2012.

34. Kim DH, Kundu JK and Surh YJ: Redox modulation of p53: mechanisms and functional significance. Mol Carcinog 50 222-234, 2011

35. Gartel AL: p21(WAF1/CIP1) and cancer: a shifting paradigm? Biofactors 35: 161-164, 2009.

36. Ocker M and Schneider-Stock R: Histone deacetylase inhibitors: signalling towards p21cip1/waf1. Int J Biochem Cell Biol 39: 1367-1374, 2007.

37. Osborne MP: Chemoprevention of breast cancer. Surg Clin North Am 79: 1207-1221, 1999.

38. Roy R, Chun J and Powell SN: BRCA1 and BRCA2: different roles in a common pathway of genome protection. Nat Rev Cancer 12: 68-78, 2011.

39. Prado A, Andrades P and Parada F: Recent developments in the ability to predict and modify breast cancer risk. J Plast Reconstr Aesthet Surg 63: 1581-1587, 2010.

40. Ohta T, Sato K and Wu W: The BRCA1 ubiquitin ligase and homologous recombination repair. FEBS Lett 585: 2836-2844, 2011.

41. Leung CC and Glover JN: BRCT domains: easy as one, two, three. Cell Cycle 10: 2461-2470, 2011.

42. Ouchi T: BRCA1 phosphorylation: biological consequences. Cancer Biol Ther 5: 470-475, 2006.

43. Kotsopoulos J and Narod SA: Androgens and breast cancer. Steroids 77: 1-9, 2012.

44. Dumitrescu RG: Epigenetic markers of early tumor development. Methods Mol Biol 863: 3-14, 2012.

45. Centrella M and McCarthy TL: Estrogen receptor dependent gene expression by osteoblasts - direct, indirect, circumspect, and speculative effects. Steroids 77: 174-184, 2012.

46. Wada-Hiraike O, Yano T, Nei T, Matsumoto Y, Nagasaka K, Takizawa S, et al: The DNA mismatch repair gene hMSH2 is a potent coactivator of oestrogen receptor alpha. Br J Cancer 92: 2286-2291, 2005.

47. Murphy LC and Leygue E: The role of estrogen receptor- $\beta$ in breast cancer. Semin Reprod Med 30: 5-13, 2012.
48. Warner M and Gustafsson JA: The role of estrogen receptor beta (ERbeta) in malignant diseases - a new potential target for antiproliferative drugs in prevention and treatment of cancer. Biochem Biophys Res Commun 396: 63-66, 2012.

49. Lamartiniere CA: Timing of exposure and mammary cancer risk. J Mammary Gland Biol Neoplasia 7: 67-76, 2002.

50. Shaik AP, Jamil K and Das P: CYP1A1 polymorphisms and risk of prostate cancer: a meta-analysis. Urol J 6: 78-86, 2009.

51. Cancel-Tassin G and Cussenot O: Prostate cancer genetics. Minerva Urol Nefrol 57: 289-300, 2005.

52. Agoulnik IU and Weigel NL: Androgen receptor action in hormone-dependent and recurrent prostate cancer. J Cell Biochem 99: 362-372, 2006.

53. Purohit A and Foster PA: Steroid sulfatase inhibitors for estrogen- and androgen-dependent cancers. J Endocrinol 212: 99-110, 2012

54. Banerjee S, Li Y, Wang Z and Sarkar FH: Multi-targeted therapy of cancer by genistein. Cancer Lett 69: 226-242, 2008.

55. Park $S$ and Choi J: Inhibition of beta-catenin/Tef signaling by flavonoids. J Cell Biochem 110: 1376-1385, 2010.

56. Power KA and Thompson LU: Can the combination of flaxseed and its lignans with soy and its isoflavones reduce the growth stimulatory effect of soy and its isoflavones on established breast cancer? Mol Nutr Food Res 51: 845-856, 2007.

57. Sánchez Y, Amrán D, Fernández C, de Blas E and Aller P: Genistein selectively potentiates arsenic trioxide-induced apoptosis in human leukemia cells via reactive oxygen species generation and activation of reactive oxygen species-inducible protein kinases (p38-MAPK, AMPK). Int J Cancer 123: $1205-1214,2008$

58. Langeberg WJ, Kwon EM, Koopmeiners JS, Ostrander EA and Stanford JL: Population-based study of the association of variants in mismatch repair genes with prostate cancer risk and outcomes. Cancer Epidemiol Biomarkers Prev 19: 258-264, 2010.

59. Lacroix-Triki M, Lambros MB, Geyer FC, Suarez PH, Reis-Filho JS and Weigelt B: Absence of microsatellite instability in mucinous carcinomas of the breast. Int J Clin Exp Pathol 27: 22-31, 2010

60. Norris AM, Woodruff RD, D'Agostino RB Jr, Clodfelter JE and Scarpinato KD: Elevated levels of the mismatch repair protein PMS2 are associated with prostate cancer. Prostate 67: 214-225, 2007.

61. Chen Y, Wang J, Fraig MM, Henderson K, Bissada NK, Watson DK, et al: Alterations in PMS2, MSH2 and MLH1 expression in human prostate cancer. Int J Oncol 22: 1033-1043, 2003.

62. Van Poppel H and Tombal B: Chemoprevention of prostate cancer with nutrients and supplements. Cancer Manag Res 3: 91-100, 2011

63. Kristal AR, Arnold KB, Neuhouser ML, Goodman P, Platz EA, Albanes D, et al: Diet, supplement use, and prostate cancer risk: results from the prostate cancer prevention trial. Am J Epidemiol 172: 566-577, 2010. 